\title{
Polymorphisms in cytochrome P450 2C19 enzyme and cessation of leflunomide in patients with rheumatoid arthritis
}

\author{
Michael D Wiese ${ }^{1,2^{*}+}$, Matthew Schnabl ${ }^{1,2+}$, Catherine O'Doherty ${ }^{1,2}$, Llewellyn D Spargo ${ }^{3}$, Michael J Sorich ${ }^{1,2}$,
} Leslie G Cleland ${ }^{3,4}$ and Susanna M Proudman ${ }^{3,4}$

\begin{abstract}
Introduction: Rational selection of disease modifying anti-rheumatic drugs in the treatment of rheumatoid arthritis (RA) has many potential advantages, including rapid disease control, reduced long-term disability and reduced overall cost to the healthcare system. Inter-individual genetic differences are particularly attractive as markers to predict efficacy and toxicity, as they can be determined rapidly prior to drug selection. The aims of this study, therefore, were to investigate the association between differences in genes associated with the metabolism, clearance and efficacy of leflunomide with its cessation in a group of rheumatoid arthritis patients who were treated with an intensive contemporary, treat-to-target approach.
\end{abstract}

Methods: This retrospective cohort study identified all individuals who received leflunomide and were enrolled in the Early Arthritis inception cohort at the Royal Adelaide Hospital between 2001 and July 2011. Inclusion criteria were age (>18) and a diagnosis of rheumatoid arthritis. Patients were excluded if a DNA sample was not available, if they withdrew from the cohort or if clinical data were insufficient. Subjects were followed for 12 months or until either another disease modifying antirheumatic drug was added or leflunomide was ceased. The following single nucleotide polymorphisms (SNPs) were determined: CYP2C19*2 (rs4244285), CYP2C19*17 (rs12248560), ABCG2 421C>A (rs2231142), CYP1A2*1F (rs762551) and DHODH 19C>A (rs3213422). The effects of variables on cessation were assessed with Cox Proportional Hazard models.

Results: Thirty-three of 78 (42.3\%) patients ceased leflunomide due to side effects. A linear trend between cytochrome P450 2C19 (CYP2C19) phenotype and leflunomide cessation was observed, with poor and intermediate metabolizers ceasing more frequently (adjusted Hazard Ratio $=0.432$ for each incremental change in phenotype, $95 \% \mathrm{Cl} 0.237$ to $0.790, P=0.006$ ). Previously observed associations between cytochrome P450 1A2 (CYP1A2) and dihydro-orotate dehydrogenase $(\mathrm{DHODH})$ genotype and toxicity were not apparent, but there was a trend for ATPbinding cassette sub-family $G$ member 2 (ABCG2) genotype to be associated with cessation due to diarrhea.

Conclusions: CYP2C19 phenotype was associated with cessation due to toxicity, and since CYP2C19 intermediate and poor metabolizers have lower teriflunomide concentrations, it is likely that they have a particularly poor risk: benefit ratio when using this drug.

\footnotetext{
* Correspondence: michael.wiese@unisa.edu.au

† Contributed equally

${ }^{1}$ Sansom Institute for Health Research, University of South Australia, Frome

Road, Adelaide, SA 5000, Australia

Full list of author information is available at the end of the article
} 


\section{Introduction}

Rheumatoid arthritis (RA) is a potentially disabling form of inflammatory arthropathy which displays both articular and systemic manifestations. Disease modifying antirheumatic drugs (DMARDs) are the cornerstone of treatment as they suppress underlying immune-mediated inflammation and joint damage and prevent long-term morbidity and mortality. Initial DMARD therapy is typically methotrexate monotherapy or a combination of DMARDs (such as 'triple therapy' with methotrexate, sulfasalazine and hydroxychloroquine [1]). In the case of resistant disease, therapy progresses to leflunomide and/ or biological DMARDs, such as tumor necrosis factor (TNF) inhibitors.

Leflunomide is an effective DMARD that was introduced for use principally as a single agent in RA resistant to better established and less expensive DMARD treatments. Nowadays, it is often combined with other DMARDs after failure of initial therapy and is considerably cheaper than biological DMARDs. In contrast to traditional single agent regimens, when leflunomide is used as an adjunct to methotrexate or the other DMARD components of 'triple therapy', lower doses are often used initially and for continuing therapy. Accordingly, there is a need to re-evaluate cessation and continuation rates when leflunomide is used as additional therapy when triple therapy alone has not achieved disease control.

Because tolerance and response to leflunomide may be influenced by polymorphisms in genes which encode proteins involved in the metabolism and clearance of leflunomide and its active metabolite, teriflunomide, we have undertaken an analysis of the effect of candidate polymorphisms on cessation/continuation rates of leflunomide. Polymorphisms were chosen based on the potential importance of proteins for which they encode on steady state teriflunomide blood levels via an effect on leflunomide metabolism (CYP2C19*2 (CYP2C19 681G $>\mathrm{A}, \mathrm{rs} 4244285)$ and $C Y P 2 C 19^{*} 17$ (CYP2C19, $608 \mathrm{C}>\mathrm{T}, \mathrm{rs} 12248560)[2,3])$ or biliary secretion of teriflunomide (ABCG2 421C>A, rs2231142) [4,5]). Polymorphisms that had been linked to leflunomide toxicity (CYP1A2*1F, (CYP1A2 163C>A, rs762551) and DHODH (DHODH 19C >A, rs3213422) [3,6,7]) were also studied. $D H O D H$ was also of interest because inhibition of the encoded protein dihydro-orotate dehydrogenase (DHODH) by teriflunomide appears to be the principal basis of the anti-inflammatory/immunosuppressive effects of leflunomide [8].

Identification of individuals with particularly unfavorable risk:benefit profiles (due to greater risk of toxicity or resistance to therapy) should permit more rational selection of DMARDs by minimizing both unwanted effects and the delay to commencement of potentially more effective, albeit more costly, therapies, such as TNF inhibitors, which ultimately should reduce long term joint damage and associated disability.

In a group of RA patients who were being treated with an intensive, contemporary approach (that is, concurrently with other conventional DMARDs, with dose modification according to a treat-to-target protocol [1]), the primary aim of this cohort study was to investigate the impact of pharmacogenomic factors on the likelihood of cessation of leflunomide after adjusting for the influence of more conventional factors.

\section{Materials and methods}

\section{RA patient population and treatment}

Patients who enrolled in the Early Arthritis inception cohort at the Royal Adelaide Hospital who had taken leflunomide between 2001 and July 2011 were considered for inclusion in this study. Other inclusion criteria were being over 18 and a diagnosis of RA according to the 1987 revised American College of Rheumatology criteria [9]. Patients were excluded from the analysis if there was no DNA sample available for analysis, if clinical records were insufficient or if they withdrew from the cohort study within the follow-up period.

Patients were treated with a standardized protocol that has been described previously [1]. Leflunomide was added after combination treatment had been up-titrated (if tolerated) to the following doses: methotrexate $(25 \mathrm{mg}$ sub-cutaneously per week), sulfasalazine (3 g orally daily) and hydroxychloroquine (400 mg orally daily). During the first three years of the cohort study, leflunomide was introduced with a loading dose (three daily doses of 100 $\mathrm{mg}$ followed by $20 \mathrm{mg}$ daily), and thereafter it was introduced at a daily dose of $10 \mathrm{mg}$. Patients were generally reviewed every six weeks while they had active disease, or every three months if disease was inactive. If treatment targets were not met while they were taking a daily leflunomide dose of $10 \mathrm{mg}$, the dose was increased to $20 \mathrm{mg}$. Data were collected for 12 months after initiation of leflunomide.

The following information was collected at baseline: age, gender, height, weight, smoking status, time since diagnosis, ethnicity, use of other DMARDs, blood pressure, full blood count, liver function tests, shared epitope, anti-cyclic citrullinated peptide (anti-CCP) antibody and rheumatoid factor titer. At each clinic visit, leflunomide dose was recorded, patients were questioned about side effects to all DMARDs and results of full blood count, blood pressure readings and liver function tests were recorded.

The duration of leflunomide was defined when the patient had either i) ceased leflunomide due to side effects or ii) was continuing treatment (for example, 12 months after initiation of leflunomide) and/or if another 
DMARD had been added. Leflunomide was deemed to have been ceased due to a side effect as determined by the patient's clinician.

Individuals who continued to take leflunomide beyond 12 months were censored at their next clinic appointment or after 400 days (whichever was earlier), and those who had another DMARD added were not considered to have ceased due to side effects and were censored on the day that the new DMARD was added and included in the analysis.

The study was approved by the research ethics committees of the Royal Adelaide Hospital and the University of South Australia. Patients gave informed written consent to their enrolment in the Royal Adelaide Hospital Early Arthritis inception cohort and for collection of blood samples for DNA extraction and analysis.

\section{Genotype evaluation}

Genomic DNA was extracted from patient peripheral blood mononuclear cells and stored at $-80^{\circ} \mathrm{C}$ until retrieval. The concentration and purity of the samples was determined using a Nanodrop (Thermo Fisher Scientific, Waltham, MA, USA). DNA was plated into a 96-well master plate at $5 \mathrm{ng} / \mu \mathrm{L}$ and stored at $-20^{\circ} \mathrm{C}$ until required.

The following SNPs were determined by TaqMan ${ }^{\circledR}$ SNP genotyping assays using an ABI 7500 Fast Real-Time PCR system (Applied Biosystems, Carlsbad, CA, USA): CYP2C19*2 (rs4244285), CYP2C19*17 (rs12248560), $A B C G 2421 \mathrm{C}>\mathrm{A}$ (rs2231142), CYP1A2*1F (rs762551) and DHODH 19C >A (rs3213422). Assays were carried out according to the manufacturer's instructions.

\section{Statistical analysis}

Statistical analyses were conducted with R Version 2.13 (The $\mathrm{R}$ foundation for statistical computing, Vienna, Austria). All analyses of genotypes were adjusted for variables that influenced the rate of cessation due to toxicity. CYP2C19 was analyzed on the basis of expected phenotype as per the system of Pare et al. [10]. CYP2C19*2 was considered the loss of function allele, while CYP2C19*17 was the gain of function allele. Briefly, poor metabolizers carry two loss of function alleles, intermediate metabolizers carry one loss of function and one wild-type allele, extensive metabolizers carry two wild-type alleles and ultra-rapid metabolizers carry either one or two gain of function alleles and no loss of function alleles. CYP2C19 unknown metabolizers carry both a loss and gain of function allele, and were excluded from this analysis. The CYP1A2*1F CC genotype and CYP2C19 poor metabolizers had a population frequency $<10 \%$, so were pooled with $C Y P 1 A 2 * 1 F$ AC genotype and CYP2C19 intermediate metabolizers respectively for analysis. Cox proportional hazard models were used to estimate the Hazard
Ratio of the association between patient characteristics and time to discontinuation of leflunomide due to side effects. The statistical significance of the Hazard Ratio was assessed using the likelihood ratio test. Proportionality of the Hazard Ratio was assessed using the goodnessof-fit statistic and by inspecting a plot of Schoenfeld residuals over time. A linear trend for effect between DHODH genotypes and CYP2C19 poor/intermediate, extensive and ultrarapid metabolizers was assessed. Other patient characteristics that influenced the risk of leflunomide discontinuation were included in a multivariate Cox proportional hazards model to adjust for potential confounding and to assess the incremental effect of adding genotype or phenotype to more conventional information. The effect of leflunomide dose was assessed using a time-dependent covariate in the Cox proportional hazards model, which indicated periods in which a leflunomide daily dose of $10 \mathrm{mg}$ or $20 \mathrm{mg}$ was being taken. Adjusted survival curves were estimated using direct adjustment [11].

\section{Results}

From the 252 individuals who had been enrolled into the Early Arthritis inception cohort up to July 2011, 84 were found to fulfil the inclusion criteria, and of these, 78 were included in the analysis. Of the six excluded individuals, three had insufficient clinical data, two withdrew from the cohort study during the follow-up period due to diagnosis of a malignant tumor and one ceased of their own volition due to a side effect unlikely to be caused by leflunomide. Of the two patients who withdrew from the cohort study, one was diagnosed with a primary colorectal tumor which was revealed by investigations undertaken after a change in bowel habit shortly after initiation of leflunomide, and the other was diagnosed with metastatic lung cancer, which was identified after 41 weeks of leflunomide treatment. The baseline characteristics of the 78 included individuals are outlined in Table 1.

During the follow-up period, a total of 50 patients (64.1\%) increased their daily leflunomide dose to $20 \mathrm{mg}$ after a median of 49 days, and 4 patients (5.1\%) required addition of another DMARD due to a sub-optimal response to leflunomide. In all, 47 individuals (60.3\%) suffered an adverse drug reaction attributable to leflunomide and $33(42.3 \%)$ ceased leflunomide due to side effects. A total of 52 side effects that were responsible for cessation are shown in Table 2, with diarrhea ( $\mathrm{n}=$ $8)$, nausea/vomiting $(n=7)$ and elevated liver transaminases $(n=6)$ being the most frequently reported. The proportion of patients still taking leflunomide over the duration of the study is shown in Figure 1.

Patient characteristics that were significantly associated with an altered rate of cessation were positive 
Table 1 Baseline patient characteristics $(n=78)$

\begin{tabular}{|c|c|}
\hline Age (years), median (IQR) & 56.4 (48 to 66.75$)$ \\
\hline Body Mass Index $\left(\mathrm{kg} / \mathrm{m}^{2}\right)$, median (IQR) & $26.6(24.4$ to 30.2$)$ \\
\hline Female & $61(78.2 \%)$ \\
\hline Ever Smoked & $47(60.3 \%)$ \\
\hline Current smokers & $22(28.2 \%)$ \\
\hline Caucasian race & $73(93.6 \%)$ \\
\hline${ }^{*}$ Continuing triple DMARD therapy & $51(65.4 \%)$ \\
\hline *Continuing methotrexate & $60(76.9 \%)$ \\
\hline${ }^{*}$ Continuing sulfasalazine & $60(76.9 \%)$ \\
\hline *Continuing hydroxychloroquine & $70(89.7 \%)$ \\
\hline Initiation of leflunomide with loading dose & $7(9.0 \%)$ \\
\hline Anti-CCP antibody $(n=77)$ & $38(49.4 \%)$ \\
\hline Rheumatoid factor & $46(59.0 \%)$ \\
\hline Shared epitope $(n=76)$ & $52(68.4 \%)$ \\
\hline Duration of disease prior to initiation of leflunomide (weeks), median (IQR) & 48 (32.3 to 92.1$)$ \\
\hline DAS28 (at start of leflunomide therapy), median (IQR) & $5.8(5.2$ to 6.5$)$ \\
\hline
\end{tabular}

*- Participants could be included in any or all of these groups.

anti-CCP antibodies at the time of RA diagnosis (Hazard Ratio $=0.43,95 \%$ CI 0.21 to $0.92, P=0.029)$ and positive rheumatoid factor at the time of RA diagnosis (Hazard Ratio $=0.49,95 \%$ CI 0.25 to $0.98, P=0.044$ ). As expected, anti-CCP antibody was strongly correlated with rheumatoid factor $(P<0.001)$. Other variables that were associated with cessation rate were current use of a $20 \mathrm{mg}$ daily dose (Hazard Ratio $=1.68,95 \%$ CI 0.79 to 3.56) and use of triple DMARD therapy at the time of leflunomide initiation (Hazard Ratio $=2.2$, 95\% CI 0.97 to 5.2), but these did not reach statistical significance $(P=0.18$ and 0.057 respectively). There was a trend for a correlation between continuation of triple therapy with anti-CCP antibodies $(P=0.079)$, but not rheumatoid factor $(P=0.97)$, so positive anti-CCP antibodies were not included in the final model.

After adjusting for current leflunomide dose, triple therapy and positive rheumatoid factor at diagnosis, CYP2C19 phenotype was significantly associated with the likelihood of cessation, with ultra-rapid metabolizers ceasing less frequently than extensive and intermediate/ poor metabolizers (Table 3 and Figure 2, Hazard Ratio = 0.432 for each incremental change in phenotype, 95\% CI 0.237 to $0.790, P=0.006$ ). As a sensitivity analysis, nonCaucasian subjects were removed from the analysis, and the resultant Hazard Ratio was 0.409 (95\% CI 0.219 to 0.762, $P=0.005)$. No association between $A B C G 2(P=$ 0.572), CYP1A2 $(P=0.954)$ or $D H O D H(P=0.993)$

Table 2 Side effects leading to cessation of leflunomide

\begin{tabular}{cc}
\hline Side effect & Number of patients reporting side effect \\
\hline Diarrhea & 8 \\
\hline Nausea/Nomiting & 7 \\
\hline Elevated transaminases* & 6 \\
\hline Shortness of breath/cough/pneumonitis & 5 \\
\hline Dizziness/fainting & 4 \\
\hline Rash & 3 \\
\hline Haematological & $3+$ \\
\hline Abdominal cramps/bloating & 3 \\
\hline Hair loss & 2 \\
\hline Fatigue & 2 \\
\hline 9 others $\neq$ & 1 each \\
\hline
\end{tabular}

* Elevated transaminases ranged from 2 to 30 times the upper limit of normal

† Includes one case each of oxidative haemolysis, neutropenia and leucopenia

₹ one each of muscle pain, blurred vision, facial flushing, mouth ulcers, taste disturbance, heavy/painful menstruation, peripheral neuropathy, loss of weight and headaches. 


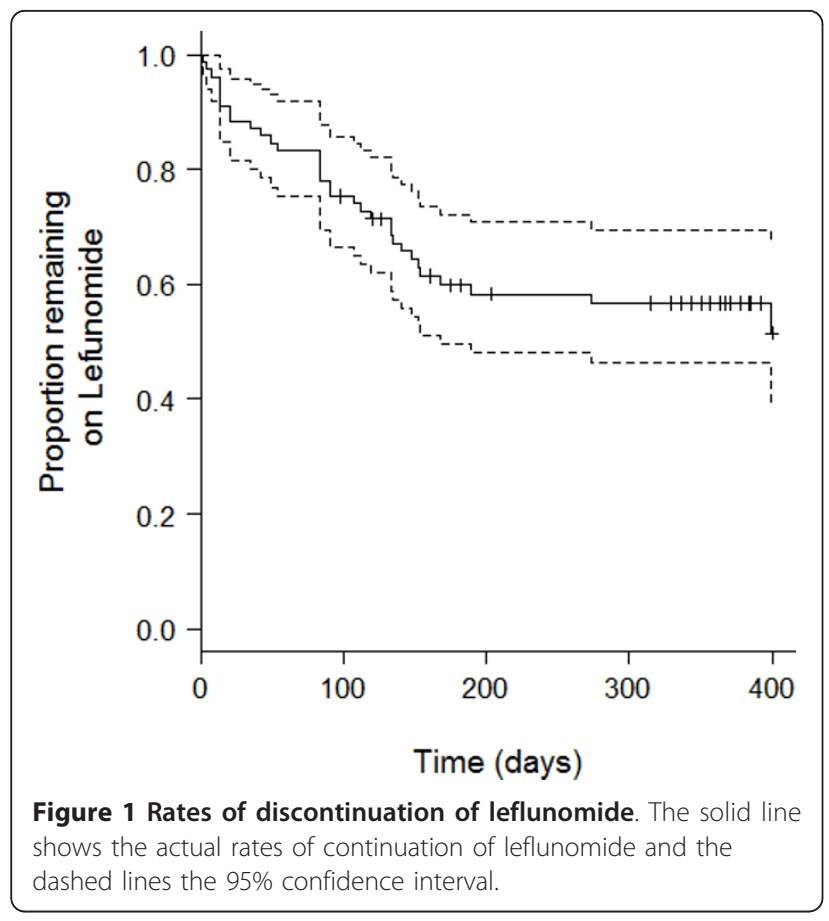

genotype and leflunomide cessation was apparent (Table 3 ), but interestingly, it was noted that while 0 of 14 who had the AC genotype at $421 \mathrm{C}>\mathrm{A} A B C G 2$ ceased leflunomide as a result of diarrhea ( $+/$ - other side effects), 8 of the 64 patients with the $421 * \mathrm{CC}$ genotype $(12.5 \%)$ ceased treatment for this reason $(P=0.198)$.

\section{Discussion}

Our results have shown a significant association between CYP2C19 phenotype and cessation of leflunomide due to toxicity in a group of patients with RA, most of whom were concurrently receiving other DMARDs.
The CYP2C19*2 loss-of-function allele (carried by poor, intermediate or unknown metabolizers) has been associated with lower teriflunomide concentration, which is thought to be due to reduced conversion of leflunomide to teriflunomide [3]. If the side effects from leflunomide were solely caused by teriflunomide, we would expect intermediate/poor metabolizers to have a lower incidence of toxicity. This was not the case in this study, and suggests either direct toxicity by leflunomide, or an alternate pathway is competing with the conversion of leflunomide to teriflunomide and results in formation of another metabolite which contributes to side effects. The latter is supported by studies of leflunomide in cultured hepatocytes, which indicated that Cytochrome P450 mediated metabolism is a prerequisite for leflunomide induced liver injury, whereas teriflunomide is directly, albeit less significantly toxic [12]. Metabolites of teriflunomide have not been observed in human plasma, but studies with human liver microsomes have shown that teriflunomide can be converted via hydroxylation to a metabolite (M1), whereas leflunomide can be converted to either teriflunomide or a different metabolite (M2, also by hydroxylation) [13,14]. If the enzymes responsible for formation of M2 are different from the well characterized conversion pathway of leflunomide to teriflunomide, reduced activity of CYP2C19 is likely to result in increased formation of M2, which may be the toxic metabolite contributing to the side effects associated with leflunomide.

While we provide no direct evidence for either the formation of M1 or M2 in vivo or toxicity associated with M2, we have demonstrated that CYP2C19 phenotypes with reduced activity were associated with higher rates of toxicity. Furthermore, since there has been an association between greater reduction in RA disease activity in patients with higher plasma teriflunomide steady state

Table 3 Association of phenotypes and genotypes with cessation due to side effects

\begin{tabular}{|c|c|c|c|}
\hline SNP & Phenotype/Genotype & $\begin{array}{c}\text { Proportion ceased due to side } \\
\text { effects (\%) }\end{array}$ & $\begin{array}{c}\text { Hazard Ratio }(95 \% \text { Confidence } \\
\text { Interval), } P \text {-value* }\end{array}$ \\
\hline \multirow[t]{3}{*}{$\begin{array}{c}\text { CYP2C19 (CYP2C19*2 (rs4244285), CYP2C19*17 } \\
\text { (rs12248560)) }\end{array}$} & $\begin{array}{l}\text { Poor/Intermediate } \\
\text { metabolizers }\end{array}$ & $8 / 15(53.3 \%)$ & 0.432 (0.237 to 0.790$), P=0.006 t$ \\
\hline & Extensive metabolizers & $18 / 39(46.2 \%)$ & \\
\hline & Ultra-rapid metabolizers & $5 / 21(23.8 \%)$ & \\
\hline \multirow[t]{2}{*}{$A B C G 2421 C>A(r s 2231142)$} & $A C$ & $6 / 14(42.9 \%)$ & 1.297 (0.527 to 3.194$), P=0.572$ \\
\hline & CC & $27 / 64(42.2 \%)$ & \\
\hline \multirow[t]{2}{*}{ CYP1A2*1F (rs762551) } & $\mathrm{AA}$ & $16 / 38(42.1 \%)$ & 1.021 (0.508 to 2.050), $P=0.954$ \\
\hline & $\mathrm{AC}+\mathrm{CC}$ & $17 / 40(42.5 \%)$ & \\
\hline \multirow[t]{3}{*}{$\mathrm{DHODH} 19 \mathrm{C}>\mathrm{A}(\mathrm{rs} 3213422)$} & $\mathrm{AA}$ & $9 / 20(45 \%)$ & $1.002(0.610$ to 1.648$), P=0.993 t$ \\
\hline & $A C$ & 16/39 (41.0\%) & \\
\hline & CC & $8 / 19(42.1 \%)$ & \\
\hline
\end{tabular}

All genotypes are in Hardy-Weinberg equilibrium. *after adjustment for dose, continuing triple therapy and positive rheumatoid factor. $\dagger$ Hazard Ratio and $P$ value represent a linear test for trend. The Hazard Ratio refers to the hazard associated with each incremental change in phenotype. 


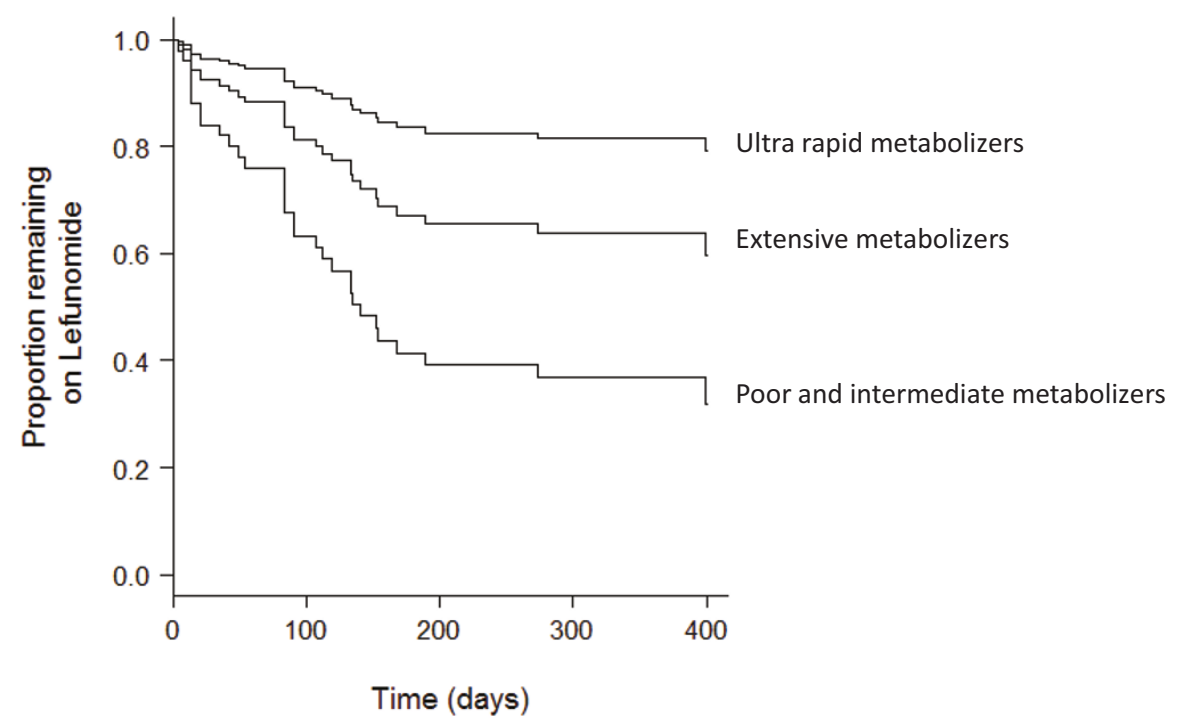

Figure 2 Cox proportional hazard model to estimate the rates of discontinuation according to CYP2C19 phenotype (Hazard Ratio = 0.432 for each incremental change in phenotype, $95 \%$ Confidence Interval 0.237 to $0.790, P=0.006$ after adjustment for current leflunomide dose, continuation of triple therapy and positive rheumatoid factor at diagnosis).

concentration $[3,15,16], C Y P 2 C 19$ intermediate/poor metabolizers may have an especially adverse risk:benefit ratio by virtue of a higher risk of cessation with side effects and a reduced likelihood of therapeutic benefit. In contrast, ultra-rapid metabolizers appear to have a particularly favorable risk:benefit ratio. This may be an important factor for clinicians to consider when they are contemplating which DMARD to prescribe their patients with RA. This finding may also explain why there has been no success in identifying a relationship between adverse drug events and teriflunomide concentration.

A number of factors are considered by both physician and patient when deciding whether to cease any medication. A novel feature of our study protocol is that a DMARD is never stopped or switched for reason of inefficacy, and the only reason to cease a DMARD is for toxicity. However, we must acknowledge that both patient and physician perceptions of side effects may be subjective, and we can not exclude the possibility that inefficacy may have influenced withdrawals. To this end, we did not observe any association between response to leflunomide after three months of treatment and CYP2C19 phenotype (data not shown), suggesting that reduced efficacy is unlikely to be responsible for increased drug cessation in CYP2C19 intermediate and poor metabolizers.

van Roon and colleagues have shown that when leflunomide was given primarily as monotherapy to long term RA patients, $29 \%$ withdrew due to an adverse event and $13 \%$ ceased due to inefficacy after a median follow-up of 317 days [17]. This compares to a withdrawal rate due to adverse events of $42.3 \%$ in our study and addition of another DMARD was required in $5.1 \%$ of patients. Since our study had a shorter mean duration from diagnosis to introduction of leflunomide (1.4 vs 9.5 years) and more frequent use of combination DMARDs, the greater efficacy and poorer tolerability observed in our cohort is to be expected and entirely consistent with this previous report [17].

The results of our study are in contrast to previous studies of leflunomide toxicity that have reported no relationship with CYP2C19 genotype, but did demonstrate an association with SNPs in CYP1A2 and DHODH [6,7]. There are a number of important differences in both study design and the participants included in our study compared to these previous reports (which were presumably performed on the same patient population) which may account for these discrepancies. First, our study was a retrospective cohort design, which has advantages over retrospective case control studies, which are prone to additional selection/recruitment bias. The previous study reported that only $19 \%$ of the group who had not ceased leflunomide were taking another DMARD (most of whom were also taking methotrexate), whereas the number who ceased leflunomide while taking other DMARDs was not reported; such a discrepancy could account for the differing rates of toxicity between genotypes, which is supported by our finding of higher cessation rates in individuals who were continuing triple therapy. Despite not accounting for these important variables, the authors adjusted for factors that did not appear to influence toxicity, such as age, body 
mass index (BMI) and disease duration. Our approach of adjusting only for variables that were seen to influence cessation due to toxicity is more robust. Finally, by comprehensively assessing information about multiple CYP2C19 genotypes, we were able to consider the entirety of the information regarding CYP2C19 activity concurrently by comparing outcomes related to $C Y P 2 C 19$ phenotype instead of each genotype separately. This is an important point of difference with previous studies, since 20 to $25 \%$ of individuals who carry a loss ("2) or gain ("17) of function allele are unknown metabolizers and should, therefore, not be considered equivalent to intermediate or ultra-rapid metabolizers respectively. As such, compared to CYP2C19 phenotypes, the effect size observed with individual genotypes is likely to be lower and significant associations are, therefore, more difficult to demonstrate.

The conversion of leflunomide to teriflunomide is mediated by CYP1A2 (in addition to CYP2C19) [2], and the immunosuppressive properties are mediated by inhibition of DHODH [8], but there is currently no biologically plausible explanation for the proposed associations of $C Y P 1 A 2$ and $D H O D H$ genotype and leflunomide toxicity $[6,7]$. The CYP1A2*1F allele occurs in the promoter region of the CYP1A2 gene (in intron 1), and is thought to be important in induction of enzymatic activity in smokers (smokers who carried one or two $\mathrm{C}$ residues had higher enzyme activity compared to those who had the AA genotype), but it does not have an effect on enzymatic activity in non-smokers [18]. If this mechanism is responsible for the higher rate of adverse events, one would expect that this SNP would only have an effect in current smokers. Given that the current smoking rate in our cohort (28\%) was higher than that reported by Bohanec Grabar et al. (18\%) [6] a more pronounced effect on toxicity would have been expected in our study, but this was not the case. Smokers who carry an AC or CC genotype are likely to be more efficient at converting lefunomide to teriflunomide, but this does not translate to higher teriflunomide concentrations [3]. Furthermore, given the association between CYP2C19 phenotype and toxicity suggests that intolerance may be due to a toxic metabolite, enhanced CYP1A2 activity (and, therefore, enhanced conversion of leflunomide to teriflunomide) is more likely to be protective of side effects. The SNP in the $D H O D H$ gene associated with toxicity by Grabar et al. (rs3413422) is associated with an amino acid substitution in the expressed protein (Lys7Gln) [7], but the effect of this on the activity of the expressed DHODH protein is not known. While there is currently no established biological plausibility to support the association of $D H O D H$ genotype and toxicity, this could change if the functional significance of this polymorphism is determined.

Although it was not statistically significant, reduced activity of the ABCG2 drug efflux pump appeared to be protective against diarrhea. This is despite individuals with reduced ABCG2 activity having higher plasma teriflunomide concentrations, which is likely due to less hepatobiliary recycling and fecal elimination [19]. Reduced secretion of teriflunomide into the gastrointestinal tract may be protective against diarrhea through reduced local effect of teriflunomide on the bowel wall.

Our study did have some weaknesses that must be acknowledged, including the retrospective design, the assessment of patient compliance was limited to direct questioning of study participants and one cannot be certain that all adverse events attributed to leflunomide were both drug related and caused by leflunomide (as opposed to one of the other DMARDs the patient was taking concurrently). Also, where a patient ceased leflunomide due to more than one side effect, no attempt was made to ascertain the relative severity of each side effect (and, therefore, the one primarily responsible for cessation). The small patient numbers also did not allow us to make definitive findings regarding the factors associated with events that occurred at low frequency within the cohort, such as the influence of $A B C G 2$ genotype on cessation due to diarrhea.

We are not aware of any previous observations that have linked side effects to any DMARD (including leflunomide) and anti-CCP antibody and rheumatoid factor positivity. However, it is notable that only a proportion of cases that conform to diagnostic or classification criteria for RA express these antibodies [9]. Accordingly, RA may be regarded as a clinical syndrome, in which a substantial proportion of cases have an autoimmune aetiology, as evidenced by the presence of anti-CCP antibodies (and rheumatoid factor with which it is strongly correlated) in serum and synovial fluid and citrullinated peptides within affected joints [20]. Cases that present with the RA syndrome but lack anti-CCP antibodies and rheumatoid factor presumably have other etiologies that, in contrast to sero-positive RA, may be less dependent upon lymphocyte proliferation to sustain a chronic, pathological antigen-specific immune response. The primary mechanism of action of teriflunomide is via inhibition of DHODH, which prevents activated T-lymphocytes from accumulating sufficient pyrimidines to support DNA synthesis, resulting in decreased cell proliferation [14]. Since methotrexate may also compromise the DHODH synthetic pathway [21], it is conceivable that sero-positive RA is particularly responsive to leflunomide, especially when given as an adjunct to methotrexate therapy. In addition to the overlapping effects of methotrexate and leflunomide on pyrimidine synthesis, sulfasalazine and leflunomide each have inhibitory effects on nuclear factor- $\kappa \mathrm{B}$ $[22,23]$. These interactive effects suggest that even lower doses of leflunomide than those used in trials hitherto 
may yield benefit and be better tolerated than current standard doses, especially in sero-positive RA.

Informed by our observations, future research should investigate whether leflunomide toxicity is mediated by a toxic metabolite, and if so, seeking to identify and quantify the putative toxic metabolite in patient plasma as a basis for defining correlations with toxicity. Also, a prospective evaluation of the factors that influence leflunomide toxicity, which also incorporates measurement of teriflunomide plasma concentrations would seem to be warranted. Such a trial would ideally have higher patient numbers through recruitment from multiple centers that utilize structured DMARD regimens. The value of $A B C G 2$ genotype in predicting diarrhea should also be assessed in a larger patient group.

\section{Conclusions}

In our group of 78 patients, most of whom were taking triple DMARD therapy in combination with leflunomide, the CYP2C19 phenotype was associated with cessation due to toxicity and altered the risk:benefit ratio when using this drug. The choice of leflunomide may, therefore, be guided further by knowledge of $C Y P 2 C 19$ phenotype.

\section{Abbreviations \\ Anti-CCP: anti-cyclic citrullinated peptide; CYP1A2: cytochrome P450 1A2; CYP2C19: cytochrome P450 2C19; DHODH: dihydro-orotate dehydrogenase; DMARD: Disease Modifying Anti-Rheumatic Drug; RA: rheumatoid arthritis; SNP: single nucleotide polymorphism; TNF: tumor necrosis factor.}

\section{Acknowledgements}

The authors would like to thank Professor Michael James for his numerous helpful suggestions throughout this study, and also Cindy Hall and Leah McWilliams for their assistance with data collection.

This work was supported by Arthritis Australia (The Barbara Cameron Memorial Grant) awarded to MDW, CO, LGC and SMP.

\section{Author details}

'Sansom Institute for Health Research, University of South Australia, Frome Road, Adelaide, SA 5000, Australia. ${ }^{2}$ School of Pharmacy and Medical Sciences, University of South Australia, Frome Road, Adelaide, SA 5000, Australia. ${ }^{3}$ Department of Rheumatology, Royal Adelaide Hospital, North Terrace, Adelaide, SA 5000, Australia. ${ }^{4}$ School of Medicine, Adelaide University, North Terrace, Adelaide, SA 5000, Australia.

\section{Authors' contributions}

MDW, MS, CO, LGC and SMP were primarily responsible for study design. Data collection was performed by MS, LDS, LGC and SMP. Data analysis was primarily performed by MDW, MS, CO and MJS. All authors helped to draft the manuscript and have read and approved the final manuscript.

\section{Competing interests}

The authors declare that they have no competing interests.

Received: 12 April 2012 Revised: 21 June 2012 Accepted: 12 July 2012 Published: 12 July 2012

\section{References}

1. Proudman SM, Keen HI, Stamp LK, Lee AT, Goldblatt F, Ayres OC, Rischmueller M, James MJ, Hill CL, Caughey GE, Cleland LG: Responsedriven combination therapy with conventional disease-modifying antirheumatic drugs can achieve high response rates in early rheumatoid arthritis with minimal glucocorticoid and nonsteroidal antiinflammatory drug use. Semin Arthritis Rheum 2007, 37:99-111.

2. Kalgutkar AS, Nguyen HT, Vaz AD, Doan A, Dalvie DK, McLeod DG, Murray JC: In vitro metabolism studies on the isoxazole ring scission in the anti-inflammatory agent lefluonomide to its active alpha-cyanoenol metabolite A771726: mechanistic similarities with the cytochrome P450catalyzed dehydration of aldoximes. Drug Metab Dispos 2003, 31:1240-1250

3. Bohanec Grabar P, Grabnar I, Rozman B, Logar D, Tomsic M, Suput D, Trdan T, Peterlin Masic L, Mrhar A, Dolzan V: Investigation of the influence of CYP1A2 and CYP2C19 genetic polymorphism on A771726 pharmacokinetics in leflunomide treated patients with rheumatoid arthritis. Drug Metab Dispos 2009, 37:2061-2068.

4. Kis E, Nagy T, Jani M, Molnar E, Janossy J, Ujhellyi O, Nemet K, HerediSzabo K, Krajcsi P: Leflunomide and its metabolite A771726 are high affinity substrates of BCRP: implications for drug resistance. Ann Rheum Dis 2009, 68:1201-1207.

5. Imai Y, Nakane M, Kage K, Tsukahara S, Ishikawa E, Tsuruo T, Miki Y, Sugimoto $Y$ : C421A polymorphism in the human breast cancer resistance protein gene is associated with low expression of Q141K protein and low-level drug resistance. Mol Cancer Ther 2002, 1:611-616.

6. Bohanec Grabar P, Rozman B, Tomsic M, Suput D, Logar D, Dolzan V: Genetic polymorphism of CYP1A2 and the toxicity of leflunomide treatment in rheumatoid arthritis patients. Eur J Clin Pharmacol 2008, 64:871-876.

7. Grabar PB, Rozman B, Logar D, Praprotnik S, Dolzan V: Dihydroorotate dehydrogenase polymorphism influences the toxicity of leflunomide treatment in patients with rheumatoid arthritis. Ann Rheum Dis 2009, 68:1367-1368.

8. Breedveld FC, Dayer JM: Leflunomide: mode of action in the treatment of rheumatoid arthritis. Ann Rheum Dis 2000, 59:841-849.

9. Arnett FC, Edworthy SM, Bloch DA, MCShane DJ, Fries JF, Cooper NS, Healey LA, Kaplan SR, Liang MH, Luthra HS, Medsger TA Jr, Mitchell DM, Neustadt DH, Pinals RS, Schaller JG, Sharp JT, Wilder RL, Hunder GG: The American Rheumatism Association 1987 revised criteria for the classification of rheumatoid arthritis. Arthritis Rheum 1988, 31:315-324.

10. Pare G, Mehta SR, Yusuf S, Anand SS, Connolly SJ, Hirsh J, Simonsen K, Bhatt DL, Fox KA, Eikelboom JW: Effects of CYP2C19 genotype on outcomes of clopidogrel treatment. N Engl J Med 2010, 363:1704-1714.

11. Ghali WA, Quan H, Brant R, van Melle G, Norris CM, Faris PD, Galbraith PD, Knudtson ML: Comparison of 2 methods for calculating adjusted survival curves from proportional hazards models. JAMA 2001, 286:1494-1497.

12. Seah $\mathrm{QM}$, New LS, Chan EC, Boelsterli UA: Oxidative bioactivation and toxicity of leflunomide in immortalized human hepatocytes and kinetics of the non-enzymatic conversion to its major metabolite, A77 1726. Drug Metab Lett 2008, 2:153-157.

13. Chan EC, New LS: In vitro metabolism of leflunomide by mouse and human liver microsomes. Drug Metab Lett 2007, 1:299-305.

14. Chan EC, New LS, Yap CW, Goh LT: Pharmaceutical metabolite profiling using quadrupole/ion mobility spectrometry/time-of-flight mass spectrometry. Rapid Commun Mass Spectrom 2009, 23:384-394.

15. van Roon EN, Jansen $T L$, van de Laar MA, Janssen M, Yska JP, Keuper $R$, Houtman PM, Brouwers JR: Therapeutic drug monitoring of A77 1726, the active metabolite of leflunomide: serum concentrations predict response to treatment in patients with rheumatoid arthritis. Ann Rheum Dis 2005, 64:569-574.

16. Chan V, Charles BG, Tett SE: Population pharmacokinetics and association between A77 1726 plasma concentrations and disease activity measures following administration of leflunomide to people with rheumatoid arthritis. Br J Clin Pharmacol 2005, 60:257-264.

17. Van Roon EN, Jansen TL, Mourad L, Houtman PM, Bruyn GA, Griep EN, Wilffert B, Tobi H, Brouwers JR: Leflunomide in active rheumatoid arthritis: a prospective study in daily practice. $\mathrm{Br} J$ Clin Pharmacol 2004, 57:790-797.

18. Sachse C, Brockmoller J, Bauer S, Roots I: Functional significance of a C>A polymorphism in intron 1 of the cytochrome P450 CYP1A2 gene tested with caffeine. Br J Clin Pharmacol 1999, 47:445-449.

19. Kim KA, Joo HJ, Park JY: Effect of ABCG2 genotypes on the pharmacokinetics of A771726, an active metabolite of prodrug leflunomide, and association of A771726 exposure with serum uric acid level. Eur J Clin Pharmacol 2011, 67:129-134. 
20. Baeten D, Peene I, Union A, Meheus L, Sebbag M, Serre G, Veys EM, De Keyser F: Specific presence of intracellular citrullinated proteins in rheumatoid arthritis synovium: relevance to antifilaggrin autoantibodies. Arthritis Rheum 2001, 44:2255-2262.

21. Ng SB, Buckingham KJ, Lee C, Bigham AW, Tabor HK, Dent KM, Huff CD, Shannon PT, Jabs EW, Nickerson DA, Shendure J, Bamshad MJ: Exome sequencing identifies the cause of a Mendelian disorder. Nat Genet 2010, 42:30-35.

22. Wahl C, Liptay S, Adler G, Schmid RM: Sulfasalazine: a potent and specific inhibitor of nuclear factor kappa B. J Clin Invest 1998, 101:1163-1174.

23. Manna SK, Aggarwal BB: Immunosuppressive leflunomide metabolite (A77 1726) blocks TNF-dependent nuclear factor-kappa B activation and gene expression. J Immunol 1999, 162:2095-2102.

doi:10.1186/ar3911

Cite this article as: Wiese et al:: Polymorphisms in cytochrome P450

2C19 enzyme and cessation of leflunomide in patients with rheumatoid arthritis. Arthritis Research \& Therapy 2012 14:R163.

\section{Submit your next manuscript to BioMed Central} and take full advantage of:

- Convenient online submission

- Thorough peer review

- No space constraints or color figure charges

- Immediate publication on acceptance

- Inclusion in PubMed, CAS, Scopus and Google Scholar

- Research which is freely available for redistribution

Submit your manuscript at www.biomedcentral.com/submit 